\title{
SOME NEW EXAMPLES OF NON-DEGENERATE QUIVER POTENTIALS
}

\author{
L. DE THANHOFFER DE VÖLCSEY AND M. VAN DEN BERGH
}

\begin{abstract}
We prove a technical result which allows us to establish the nondegeneracy of potentials on quivers in some previously unknown or non-obvious cases.

Our result applies to certain McKay quivers and also to potentials derived from geometric helices on Del Pezzo surfaces.
\end{abstract}

\section{INTRODUCTION}

In [10] Derksen, Weyman and Zelevinsky describe how to "mutate" at a vertex a pair $(Q, W)$ consisting of a quiver $Q$ and a potential $W \in(k Q)^{\wedge} /\left[(k Q)^{\wedge},(k Q)^{\wedge}\right]$. This construction produces a new such pair $\left(Q^{\prime}, W^{\prime}\right)$. The Jacobian (or Ginzburg) algebras of the pairs $(Q, W),\left(Q^{\prime}, W^{\prime}\right)$ share many homological properties $[7,11,13]$.

One peculiarity of the mutation process is that it is only defined if the vertex is not incident to a loop or two-cycle. Even if all vertices in $Q$ have this property then this is not necessarily the case for $Q^{\prime}$. If the property of having no loops or two-cycles persists under iterated mutations then we say that $(Q, W)$ (or $W$ ) is non-degenerate.

In this paper we give a technical result (Theorem 3.1) which allows us to establish the non-degeneracy of potentials in some previously unknown or non-obvious cases. Our theorem implies for example that potentials derived from geometric helices on Del Pezzo surfaces (see below) are non-degenerate. In this way we recover part of the main result of [6].

Our theorem also applies to the McKay quiver associated to a group $G \subset \operatorname{SL}(V)$ where $\operatorname{dim} V=3$ and $G$ acts freely on $V^{*}-\{0\}$ (this implies that $G$ is cyclic). See Corollary 4.1 .2 below.

In fact the original motivation of our work was an explicit example considered by Iyama and Reiten in [11] where they mention that they do not know if the mutations of the associated McKay quiver do not create loops or two-cycles.

1991 Mathematics Subject Classification. 16T30.

Key words and phrases. Quiver potentials, mutations.

The second author is a senior researcher at the FWO. 
Assume that $Q$ is the McKay quiver of the pair $\left(\mathbb{Z} / 5 \mathbb{Z}, k x_{1}+k x_{2}+k x_{3}\right)$ where the generator of $\mathbb{Z} / 5 \mathbb{Z}$ acts diagonally by $\left(\xi, \xi^{2}, \xi^{2}\right)$. The quiver $Q$ looks as follows

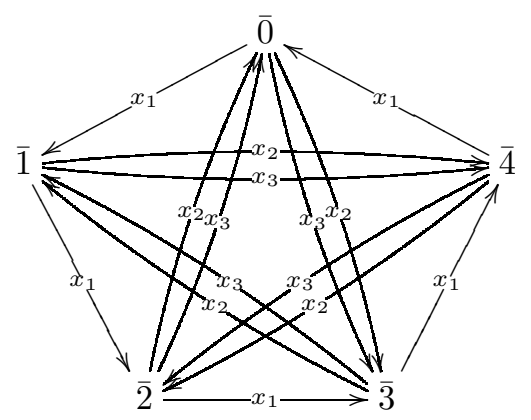

The potential $W$ is the signed sum of all three-cycles containing $x_{1}, x_{2}$ and $x_{3}$. The Jacobian algebra of $(Q, W)$ is the skew group ring $k\left[\left[x_{1}, x_{2}, x_{3}\right]\right] \# \mathbb{Z} / 5 \mathbb{Z}$. Our main theorem implies that $W$ is non-degenerate.

On the other hand if $G$ does not act freely on $V^{*}-\{0\}$ then the associated potential will generally be degenerate. See Example 4.1.3 below.

The main technical tool in this paper is the lifting of mutations to the graded setting (see [1]).

Throughout all quivers are finite. $k$ is a base field.

\section{Graded And ungraded mutations}

Let $Q$ be a quiver. A potential on $Q$ is an element $W \in(k Q)^{\wedge} /\left[(k Q)^{\wedge},(k Q)^{\wedge}\right]$ containing no paths of length one or zero. We write $W$ as a sum of oriented cycles in $Q$. The corresponding Jacobian algebra is defined in the usual way

$$
\mathcal{P}(Q, W)=(k Q)^{\wedge} /\left(\partial_{a} W\right)_{a}
$$

The space of paths of length zero in $k Q$ will be denoted by $R$. This is a semisimple $k$-algebra.

A potential is said to be reduced if it contains no two-cycles. Such two-cycles lead to relations which express some arrows in terms of others and hence such arrows can be eliminated in the Jacobian algebra. This observation is refined in the splitting theorem $[10$, Thm 4.6] which asserts that $(Q, W)$ is "right equivalent" (see [10, Def. 4.2]) to a direct sum decomposition

$$
(Q, W) \sim\left(Q^{\text {red }}, W^{\text {red }}\right) \oplus\left(Q^{\text {triv }}, W^{\text {triv }}\right)
$$

where $W^{\text {red }}$ is reduced and $W^{\text {triv }}$ contains only two-cycles and its associated Jacobian algebra is equal to $R$.

The decomposition theorem implies in particular

$$
\mathcal{P}(Q, W) \cong \mathcal{P}\left(Q^{\text {red }}, W^{\text {red }}\right)
$$

If $A$ is an abelian group then we say that $Q$ is $A$-graded if we have assigned an $A$-degree $|a|$ to all arrows $a$ in $Q$. If $W$ is homogeneous of degree $r \in A$ the partially completed Jacobian algebra

$$
\mathcal{P}^{\mathrm{gr}}(Q, W)=(k Q)^{\mathrm{gr}} \wedge /\left(\partial_{a} W\right)_{a}
$$

(where we complete only with respect to sequences of paths having ascending length but constant degree), is naturally an $A$-graded algebra. It is observed in [1] that 
the graded analogue of the splitting theorem holds and hence the decomposition (2.1) can be performed on the graded level.

The following lemma is very useful.

Lemma 2.1. Let $Q^{\prime}$ be obtained from $Q$ by repetively deleting pairs of arrows $i \stackrel{a}{\rightarrow} j \stackrel{b}{\rightarrow} i$ with $i \neq j$ and $|a|+|b|=r$. If $Q^{\prime}$ contains a two-cycle then so does $Q^{\text {red }}$.

Proof. By the degree constraint on $W$ we can only eliminate two-cycles of the form $a b$ with $|a|+|b|=r$. This implies the result.

Assume that $Q$ does not have loops or two-cycles and let $i$ be a vertex of $Q$. The mutation $\mu_{i}(Q, W)=\left(Q^{\prime}, W^{\prime}\right)$ at $i$ is defined in [10] as follows.

- For any sequence of arrows $u \stackrel{a}{\rightarrow} i \stackrel{b}{\rightarrow} v$ we add an arrow $u \stackrel{[b a]}{\longrightarrow} v$.

- All arrows $a$ starting or ending in $i$ are replaced with opposite arrows $a^{*}$.

We have

$$
W^{\prime}=[W]+\sum b^{*} a^{*}[a b]
$$

where $[W]$ is obtained from $W$ by replacing all compositions $b a$ through the vertex $i$ by the new arrows $[b a]$. Following $[10]$ we put $\tilde{\mu}_{i}(Q, W)=\left(Q^{\prime \text { red }}, W^{\prime \text { red }}\right)$. The operations $\tilde{\mu}_{i}$ is an operation on right equivalence classes of quivers with potential (see [10, Thm 5.2]).

Now assume that $Q$ is in addition $A$-graded and that $W$ is homogeneous of degree $r$. Then we make $Q^{\prime}$ into a $A$-graded quiver by fixing the degrees of the arrows as follows $[1]$

- Arrows which are both in $Q$ and $Q^{\prime}$ do not change degrees.

- $|[a b]|=|a|+|b|$.

- $\left|a^{*}\right|=-|a|+r$ if $a$ ends in $i$.

- $\left|a^{*}\right|=-|a|$ if $a$ starts in $i$.

With this grading $W^{\prime}$ is homogeneous of degree $r$. If we perform mutation on the graded level we write $\mu_{i}^{\mathrm{gr}}(Q, W)=\left(Q^{\prime}, W^{\prime}\right)$ and $\tilde{\mu}_{i}^{\mathrm{gr}}(Q, W)=\left(Q^{\prime \text { red }}, W^{\prime \text { red }}\right)$. Such graded mutations are compatible with forgetting the grading.

\section{MAIN THEOREM}

Let $S=\bigoplus_{n>0} S_{n}$ be a commutative noetherian $\mathbb{Z}$-graded ring and let $\Lambda$ be a graded $S$-order (i.e. $\Lambda$ is a graded $S$-algebra, finite as a module over $S$ ). We will say that $\Lambda / S$ is almost Azumaya if for all $P \in \operatorname{Spec} S$ such that $P \not \supset S_{\geq 1}$ the algebra $\Lambda_{P}$ is Azumaya over $S_{P}$. This implies in particular that for any non-zero idempotent $e \in \Lambda_{0}$ the quotient $\Lambda / \Lambda e \Lambda$ is a finite $S_{0}$-module. The latter property is in fact the only one we will use below.

Theorem 3.1. Assume the following assumptions hold

(1) $(Q, W)$ is a $\mathbb{Z}$-graded connected quiver with a reduced homogeneous potential $W$ of degree $r$.

(2) $Q$ has at least three vertices.

(3) Put $\Lambda=\mathcal{P}^{\mathrm{gr}}(Q, W)$. Then $\operatorname{dim} \Lambda_{i}<\infty$ for all $i$ and $\operatorname{dim} \Lambda_{i}=0$ for $i \ll 0$.

(4) $\Lambda$ is a 3-dimensional $C Y$-order over a noetherian center which is almost Azumaya. 
(5) The zeroeth Hochschild homology $\mathrm{HH}_{0}(\Lambda) \stackrel{\text { def }}{=} \Lambda /[\Lambda, \Lambda]$ of $\Lambda$ contains no homogeneous elements with degree lying in the interval $[1, r / 2]$.

Then $Q$ has no loops or two-cycles.

Proof. Assume first that $Q$ contains a loop $i \stackrel{a}{\rightarrow} i$ and let $e$ be the sum of the idempotents corresponding to the vertices different from $i$. Let $Q_{0}$ be the subquiver of $Q$ consisting of all loops at $i$. Put $\bar{\Gamma}=\Gamma / \Gamma e \Gamma$. Then $\bar{\Gamma}$ is the graded Jacobian algebra $\mathcal{P}^{\text {gr }}\left(Q_{0}, W_{0}\right)$ where $W_{0}$ is obtained from $\bar{W}$ by dropping all cycles passing through vertices other than $i$.

If $W_{0}=0$ then $\bar{\Gamma}$ is infinite dimensional which is impossible by the discussion preceding the theorem. Hence $W_{0}$ is a sum of terms of degree $r$ which are products of loops

$$
a_{1} \cdots a_{n}
$$

with $n \geq 3$ (since $W_{0}$ is reduced). Assume that (3.1) is the shortest such term. Then there must be some $a_{i}$ which has degree $\leq r / 3 \leq r / 2$. As the paths occurring in the relations of $\Gamma / \Gamma e \Gamma$ are products of at least two arrows we have $a_{i} \neq 0$ in $\Gamma / \Gamma e \Gamma$. Furthermore it is also clear that $a_{i}$ is not in the image of $\left[k Q_{0}, k Q_{0}\right]$. Thus $a_{i}$ represents a non-zero element of $\mathrm{HH}_{0}(\bar{\Gamma})$. Since there is a surjection $\mathrm{HH}_{0}(\Gamma) \rightarrow$ $\mathrm{HH}_{0}(\bar{\Gamma})$, we obtain a non-trivial element of $\mathrm{HH}_{0}(\Gamma)$ as well. Since $\left|a_{i}\right| \leq r / 2$ we obtain from the hypotheses that $\left|a_{i}\right| \leq 0$. But then $a_{i}$ must be nilpotent in $\Gamma$ and hence by [15] $a_{i}$ is a sum of commutators, which is a contradiction.

We now assume that $Q$ has no loops. The proof in the case of a two-cycle $i \rightarrow j \rightarrow i$ is similar and we only sketch it. Let $Q_{0}$ be the subquiver of $Q$ consisting of all arrows between $i$ and $j$ and vice versa and let $e$ be the sum of the idempotents corresponding to the vertices different from $i$ or $j$. Put $\bar{\Gamma}=\Gamma / \Gamma e \Gamma$. Then $\bar{\Gamma}=$ $\mathcal{P}^{\text {gr }}\left(Q_{0}, W_{0}\right)$ where $W_{0}$ is obtained from $W$ by dropping all cycles passing through vertices other than $i$ or $j$.

The case $W_{0}=0$ is again impossible. Hence $W_{0}$ is a sum of terms of degree $r$ which are products of two-cycles

$$
a_{1} b_{1} a_{2} b_{2} \cdots a_{n} b_{n}
$$

with $i \stackrel{a_{l}}{\longrightarrow} j$ and $j \stackrel{b_{m}}{\longrightarrow} i$ with $n \geq 2$. Hence one of the two-cycles $a_{i} b_{i}$ must have degree zero. Since this time the relations have degree $\geq 3$ we have $a_{i} b_{i} \neq 0$. This gives a non-trivial element in $\mathrm{HH}_{0}(\Gamma)$ which must have degree $\leq 0$ by the hypotheses. Thus $a_{i} b_{i}$ is nilpotent and hence is a sum of commutators which is a contradiction.

Proposition 3.2. Let $(Q, W)$ be as in Theorem 3.1 and let $i$ be a vertex of $Q$. Then up to right equivalence we may assume that $\tilde{\mu}_{i}(Q, W)=\left(Q^{\prime}, W^{\prime}\right)$ also satisfies the conditions of Theorem 3.1. In particular $Q^{\prime}$ has no loops or two-cycles.

Proof. Since ungraded and graded mutations are compatible and since the decomposition (2.1) can be performed at the graded level, we can up to right equivalence, assume that $Q^{\prime}$ is graded and $W^{\prime}$ is homogeneous.

It has been proved in $[7$, Thm 5.2$]$ and in $[13, \S 6]$ that for Jacobian algebras which are Calabi-Yau of dimension three, Derksen-Weyman-Zelevinsky mutations correspond to Iyama-Reiten mutations. With some work these proofs can be carried over to the graded context. Hence in particular we obtain that $\Gamma=\mathcal{P}^{g r}\left(Q^{\prime}, W^{\prime}\right)$ 
is 3-CY. Furthermore $Z(\Gamma)=Z(\Lambda), \Gamma$ is almost Azumaya over its center and $\Gamma$ is derived equivalent to $\Lambda$ [11]. Finally by the derived invariance of Hochschild homology we have an isomorphism of graded vector spaces

$$
\Lambda /[\Lambda, \Lambda]=\mathrm{HH}_{0}(\Lambda)=\mathrm{HH}_{0}(\Gamma)=\Gamma /[\Gamma, \Gamma]
$$

Corollary 3.3. If $(Q, W)$ is as in Theorem 3.1 then $(Q, W)$ is non-degenerate.

Proof. This is clear from Proposition 3.2.

\section{EXAmples AND COUnTEREXAMPles}

4.1. Skew group rings. In this section we assume that $k$ is algebraically closed of characteristic zero. Let $V$ be a three dimensional vector space and let $G$ be a finite subgroup of $\operatorname{SL}(V)$ of order $n$. Then it is well-known that the skew group algebra $\Lambda=S V \# G$ is 3-Calabi-Yau. Furthermore $\Lambda$ is graded Morita equivalent to a graded Jacobian algebra $\operatorname{P}^{\text {gr }}(Q, W)$ where $Q$ is the McKay quiver of $(G, V)$ and $W$ has degree three. The McKay quiver is the quiver with vertices the irreducible representations $\left(V_{i}\right)_{i}$ of $G$ and arrows $i \rightarrow j$ with multiplicity $p$ if $V_{j}$ occurs $p$ times in $V \otimes V_{i}$. We refer to [3, Thm 3.2] for a detailed discussion on how to construct the potential.

In the case of a cyclic group $G \cong \mathbb{Z} / n \mathbb{Z}$, the situation is particularly simple. The irreducible representations of $G$ are given by characters ("weights"). After the choice of a primitive $n$-th root of unity $\xi$ a character $\chi$ may be identified with an element $\bar{a}$ of $\mathbb{Z} / n \mathbb{Z}$ through the rule $\chi_{\bar{a}}(\bar{m})=\xi^{a m}$.

Let $\bar{a}_{1}, \bar{a}_{2}, \bar{a}_{3}$ be the weights of $V$. The fact that $G \subset \mathrm{SL}(V)$ is equivalent to $\sum_{i} \bar{a}_{i}=\overline{0}$. The vertices of the McKay quiver of $(G, V)$ are indexed by the elements of $\mathbb{Z} / n \mathbb{Z}$. If $\bar{l} \in \mathbb{Z} / n \mathbb{Z}$ then there are three arrows $x_{1}, x_{2}, x_{3}$ leaving $\bar{l}$ and ending respectively in $\bar{l}+\bar{a}_{1}, \bar{l}+\bar{a}_{2}, \bar{l}+\bar{a}_{3}$. The potential is given by

$$
W=\sum \pm x_{i_{1}} x_{i_{2}} x_{i_{3}}
$$

where the sum runs over all three-cycles such that $\left\{i_{1}, i_{2}, i_{3}\right\}=\{1,2,3\}$ and the sign is positive iff $\left(i_{1}, i_{2}, i_{3}\right)$ is equal to $(1,2,3)$, up to cyclic permutation.

Lemma 4.1.1. The hypotheses of Theorem 3.1 hold for $(Q, W)$ if and only if $G \cong \mathbb{Z} / n \mathbb{Z}$ is cyclic and acts with weights $\left(\bar{a}_{i}\right)_{i}$ on $V$ such that $\operatorname{gcd}\left(n, a_{i}\right)=1$.

Proof. Assume that the hypotheses for Theorem 3.1 hold. It is well-known that $\Lambda$ is almost Azumaya over its center if and only if $S V^{G}$ has an isolated singularity which is equivalent to $G$ acting freely on $V^{*}-\{0\}$ and hence that $G$ is cyclic (see [14]). The fact that $G$ acts freely is equivalent to the stated condition on weights.

Conversely assume that $G$ is cyclic and acts with weights relatively prime to $n$. We verify the hypotheses of Theorem 3.1: we have already mentioned (1). (2) follows from the fact that $\mathbb{Z} / 2 \mathbb{Z}$ cannot act freely on $V-\{0\}$ (one weight must be zero). (3) is clear. (4) has been mentioned previously. It remains to check (5). We must show $\mathrm{HH}_{0}(\Lambda)_{1}=0$. If $\mathrm{HH}_{0}(\Lambda)_{1} \neq 0$ then $Q$ must have a loop. This implies that one of the weights is zero and thus not relatively prime to $n$. Contradiction!

We obtain:

Corollary 4.1.2. Assume that $Q$ is the McKay quiver of a cyclic group $\mathbb{Z} / n \mathbb{Z}$ acting with weights $\bar{a}_{1}, \bar{a}_{2}, \bar{a}_{3}$ such that $\operatorname{gcd}\left(a_{i}, n\right)=1$ and $\sum_{i} \bar{a}_{i}=0$. Let $W$ be the potential (4.1). Then $W$ is non-degenerate. 
On the other hand the hypotheses for Corollary 4.1.2 can not be weakened as shown by the following example:

Example 4.1.3. Let $G=\mathbb{Z} / 6 \mathbb{Z}$ and assume that $G$ acts with weights $(\overline{2}, \overline{5}, \overline{5})$. Since $2 \mid 6$ the hypotheses for Corollary 4.1.2 do not hold.

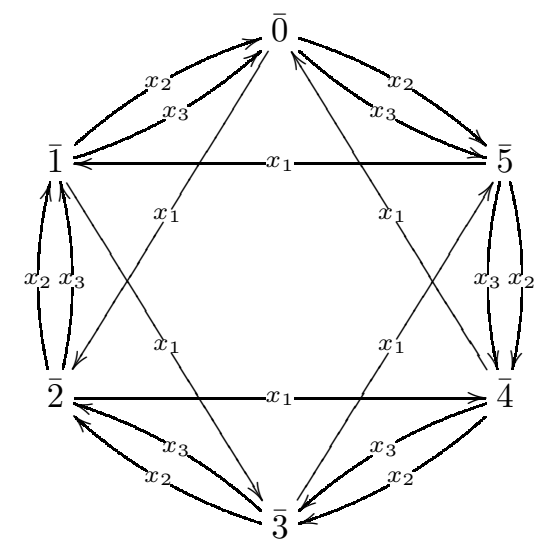

We note that the McKay quiver of a cyclic groups is naturally $\mathbb{Z}^{3}$-graded. It is convenient to use the grading by the monomials in $x_{1}, x_{2}, x_{3}$ such that $\left|x_{i}\right|=x_{i}$. Then $W$ is homogeneous of degree $x_{1} x_{2} x_{3}$. With these conventions we see that performing a mutation at the vertex $\overline{0}$ results in a two-cycle $\left[x_{1} x_{1}\right] x_{1}$ between vertices $\overline{2}$ and $\overline{4}$. This two-cycle has degree $x_{1}^{3}$ and cannot be eliminated for degree reasons (see Lemma 2.1).

4.2. Del Pezzo surfaces. Let $Y$ be a Del-Pezzo surface and let $\left(E_{i}\right)_{i=1, \ldots, n}$ be a full exceptional collection on $Y$. Put $\mathbb{E}=\bigoplus_{i=1}^{n} E_{i}$ and define $A(\mathbb{E})=\operatorname{End}(\mathbb{E})$. Assume that

$$
\mathbb{H}(E)=\left(E_{i}\right)_{i \in \mathbb{Z}}=\left(\ldots, \omega_{Y} \otimes E_{n}, E_{1}, \ldots, E_{n}, \omega_{Y}^{-1} \otimes, E_{1}, \ldots\right)
$$

is a geometric helix $($ see $[4,6])$. I.e. every slice $\left(E_{i+1}, \ldots, E_{i+n}\right)$ is an exceptional collection and furthermore

$$
\forall i<j, \forall k>0: \operatorname{Ext}^{k}\left(E_{i}, E_{j}\right)=0
$$

The rolled up helix algebra is the $\mathbb{Z}$-graded ring

$$
B(\mathbb{H})=\bigoplus_{k \in \mathbb{Z}} \operatorname{Hom}\left(\mathbb{E}, \omega_{Y}^{-k} \otimes \mathbb{E}\right)
$$

with obvious multiplication.

Theorem 4.2.1. $B(\mathbb{H})$ is an $\mathbb{N}$-graded Jacobian algebra derived from a graded super potential of degree one which is Calabi-Yau of dimension three (as ungraded algebra) and which is almost Azumaya over its center.

This result is folklore among physicists. It follows from the work by Ed Segal in [20] although it is not really stated explicitly there. The fact that $B(\mathbb{H})$ is CalabiYau of dimension dimension three is $[6$, Thm 3.6]. The fact that $B(\mathbb{H})$ is Jacobian follows from [21] and probably also from the proof of [2, Thm 3.1] although the formulation this theorem is for quivers whose arrows have degree one.

We give for the convenience of the reader an independent proof of this theorem in Appendix A based on some results from [12]. 
Theorem 4.2.2. Let $(Q, W)$ be such that $W$ is reduced and $\mathcal{P}^{\text {gr }}(Q, W)=B(\mathbb{H})$. Then $(Q, W)$ satisfies the hypotheses of Theorem 3.1.

In particular it follows from Corollary 3.3 that $(Q, W)$ is non-degenerate. This is a consequence of $[6, \mathrm{Thm}$ 1.7]. In loc. cit. the authors deduce this fact from their result that a mutation of $B(\mathbb{H})$ is always of the form $B\left(\mathbb{H}^{\prime}\right)$ for another geometric helix $\mathbb{H}^{\prime}$.

Proof of Theorem 4.2.2. (1) is true with $r=1$. (2) is true since $\operatorname{rk} K_{0}(\operatorname{coh}(Y)) \geq 3$. (3) is obvious. (4) follows from Theorem 4.2.1. (5) is vacuous since the potential has degree one.

4.3. Deformed preprojective algebras. In this section we given an easy example which shows that hypothesis (5) of Theorem 3.1 cannot be omitted. For simplicity we assume that $k$ is algebraically closed of characteristic zero.

Let $Q=\left(Q_{0}, Q_{1}\right)\left(Q_{0}=\right.$ vertices, $Q_{1}=$ edges $)$ be an extended Dynkin quiver and let $\tilde{Q}$ be its associated double quiver. That is, for every arrow $a \in Q_{1}$ we adjoin an arrow $a^{*}$ with the opposite orientation. By virtue of the construction every arrow in $\tilde{Q}$ is part of a two-cycle.

For $\left(\lambda_{i}\right)_{i} \in k^{Q_{0}}$ we define a corresponding central extension of the preprojective algebra as follows

$$
\Pi^{\lambda}=k[t] \tilde{Q} /\left(\sum_{a \in Q_{1}}\left[a, a^{*}\right]-\sum_{i \in Q_{0}} \lambda_{i} t e_{i}\right)
$$

where as usual $e_{i}$ is the idempotent corresponding to $i \in Q_{0}$. This algebra (or at least its principal representation space) is introduced in [8]. It has a natural grading with the arrows in $\tilde{Q}$ having degree one and $\operatorname{deg} t=2$. Let $\bar{Q}$ be obtained from $\tilde{Q}$ by adjoining an additional loop at every vertex. Then $\Pi^{\lambda}$ is the graded Jacobian algebra of $(\bar{Q}, \bar{W})$ with

$$
\bar{W}=\left(\sum_{i \in Q_{0}} t_{i}\right) \cdot\left(\sum_{a \in Q_{1}}\left[a, a^{*}\right]\right)-\sum_{i \in Q_{0}} \frac{1}{2} \lambda_{i} t_{i}^{2}
$$

where $t_{i}=t e_{i}$ is the added loop at $i$. This induces the potential [8, eq. (3.3)] on representation spaces.

If all $\lambda_{i}$ are non-zero then the loops can be eliminated and $\Pi^{\lambda}$ becomes the Jacobian algebra of $(\tilde{Q}, W)$ with

$$
W=\sum_{a, b \in Q_{1}, i \in Q_{0}} \frac{1}{\lambda_{i}} e_{i}\left[a, a^{*}\right]\left[b, b^{*}\right]
$$

Let 0 be the extending vertex of $Q$ (i.e. $Q-\{0\}$ is a Dynkin diagram). Following [9] we say that $\alpha \in \mathbb{N} Q_{0}$ is a Dynkin root if $\alpha$ is a root such that $\alpha_{0}=0$.

The following proposition collects some basic facts about $\Pi^{\lambda}$.

Proposition 4.3.1. Let $\delta \in k Q_{0}$ be the indecomposable imaginary root and assume that $\delta \cdot \lambda \stackrel{\text { def }}{=} \sum_{i \in Q_{0}} \delta_{i} \lambda_{i}=0$. Assume in addition that for all Dynkin roots $\alpha$ one has $\alpha \cdot \lambda \neq 0$ (this is implies $\lambda_{i} \neq 0$ for all $i$ ). Then $\Pi^{\lambda}$ is a 3-CY order which is almost Azumaya over a noetherian center. 
Proof. Most of this is stated in [18] and proved using results from [9]. Let $G \subset \mathrm{Sl}_{2}(k)$ be the Kleinian group whose McKay quiver is $\tilde{Q}$. The vertices of $\tilde{Q}$ correspond to the irreducible representations of $G$ and in particular the extending vertex corresponds to the trivial representation.

For $i \in Q_{0}$ let $f_{i}$ be the central idempotent in $k G$ corresponding to the irreducible $G$-representation indexed by $i$. Then $\Pi^{\lambda}$ is Morita equivalent to another ring $A^{\lambda}$ (a symplectic reflection algebra)

$$
k[t]\langle x, y\rangle \# G /\left(x y-y x-t \sum_{i \in Q} \frac{\lambda_{i}}{\delta_{i}} f_{i}\right)
$$

and under this equivalence the augmentation idempotent $e \in k G$ corresponds to $e_{0}$ (see [9, Thm 3.4]). The condition $\delta \cdot \lambda=0$ translates into the fact that $\sum_{i \in Q} \frac{\lambda_{i}}{\delta_{i}} f_{i}$ has trace zero for the regular representation. In other words its image under the projection on the identity component $k G \rightarrow k$ is zero.

Based on the fact that gr $A^{\lambda}=k[t][x, y] \# G$ it is deduced in $[18, \S 2.2]$ that $A^{\lambda}$ is a $k[t]$-flat prime noetherian maximal order which is Auslander-Gorenstein and Cohen-Macaulay of GK and global dimension three. In a similar way $C^{\lambda}$ is a noetherian integral domain of GK dimension 3 which is a flat (graded) deformation of the hypersurface ring $k[x, y]^{G}$. In particular $C^{\lambda}$ is also Gorenstein. Furthermore one has

$$
A^{\lambda} \cong \operatorname{End}_{C^{\lambda}}\left(A^{\lambda} e\right)
$$

In [18, Cor. 3.5] it is shown that $C^{\lambda}$ is commutative and that $C^{\lambda}$ is in fact isomorphic to the center of $A^{\lambda}$. Finally it then follows from [18, Prop. 3.2] that $A^{\lambda}$ is a noncommutative crepant resolution of its center [22]. This implies that $A^{\lambda}$ is 3 -CY. All these results carry over to $\Pi^{\lambda}$ by Morita equivalence.

In particular (4.2) becomes

$$
\Pi^{\lambda} \cong \operatorname{End}_{e_{0} \Pi^{\lambda} e_{0}}\left(\Pi^{\lambda} e_{0}\right)
$$

with $e_{0} \Pi^{\lambda} e_{0} \cong C^{\lambda}$. To prove that $\Pi^{\lambda}$ is almost Azumaya it suffices to prove that $S=\Pi^{\lambda} / \Pi^{\lambda} e_{0} \Pi^{\lambda}$ is finite dimensional. According to [9, Cor. 9.6] $S / S(t-1)=0$. Since $S$ is graded this is implies that $S_{t}=0$ (this is a statement purely about graded $k[t]$-modules). Since $S$ is finitely generated as $\Pi^{\lambda}$-module it follows that $t^{n} S=0$ for some $n$. On the other hand we also have that $S / t S$ is finite dimensional since for example $\Pi^{\lambda} / t \Pi^{\lambda}$ is the preprojective algebra of $Q$ which is Morita equivalent to $k[x, y] \# G$ and the latter is almost Azumaya (see [19]). It now follows that $S$ is finite dimensional.

So if $Q$ has three or more vertices (i.e. $\left.Q \neq A_{1}, A_{2}\right)$ and $\lambda$ is as in Proposition 4.3.1 then $\Pi^{\lambda}$ satisfies all conditions of Theorem 3.1 except (5). Indeed the degree of the potential is four and $\Pi^{\lambda} /\left[\Pi^{\lambda}, \Pi^{\lambda}\right]$ contains elements of degree two (the twocycles in $\tilde{Q})$.

And since $\tilde{Q}$ has two-cycles, the conclusion of Theorem 3.1 does indeed not hold. So hypothesis (5) cannot be deleted from the statement of Theorem 3.1.

\section{Appendix A. Potentials associated to Del Pezzo surfaces}

Let the notations be as in $\S 4.2$. In this appendix we will give an independent proof of Theorem 4.2.1. We keep the setting of the theorem although with trivial 
changes most of the proof applies to arbitrary Fano varieties. Our arguments are related to those appearing in recent papers by Minamoto and Mori [16, 17].

Lemma A.1. $B(\mathbb{H})$ is the derived tensor algebra of $B(\mathbb{H})_{1}$ over $B(\mathbb{H})_{0}=A(\mathbb{E})$.

Proof. For $k<0$ we have

$$
\operatorname{Hom}\left(\mathbb{E}, \omega_{Y}^{-k} \otimes \mathbb{E}\right)=\operatorname{Ext}^{2}\left(\mathbb{E}, \omega_{Y}^{k+1} \otimes \mathbb{E}\right)^{*}=0
$$

For $k \geq 0$ we have

$$
\operatorname{Hom}\left(\mathbb{E}, \omega_{Y}^{-k} \otimes \mathbb{E}\right)=R \operatorname{Hom}\left(\mathbb{E}, \omega_{Y}^{-k} \otimes \mathbb{E}\right)
$$

For $k, l \geq 0$ we have to prove that the canonical map

$$
\mathrm{RHom}\left(\mathbb{E}, \omega_{Y}^{-k} \stackrel{L}{\otimes} \mathbb{E}\right) \stackrel{L}{\otimes}_{A(\mathbb{E})} \mathrm{RHom}\left(\mathbb{E}, \omega_{Y}^{-l} \otimes \mathbb{E}\right) \rightarrow \mathrm{RHom}\left(\mathbb{E}, \omega_{Y}^{-k-l} \otimes \mathbb{E}\right)
$$

is an isomorphism

One verifies that this is equivalent to showing that the composition map

$$
\mathrm{RHom}\left(\mathbb{E}, \omega_{Y}^{-k} \stackrel{L}{\otimes} \mathbb{E}\right) \stackrel{L}{\otimes}_{A(\mathbb{E})} \mathrm{RHom}\left(\omega_{Y}^{l} \otimes \mathbb{E}, \mathbb{E}\right) \rightarrow \mathrm{RHom}\left(\omega_{Y}^{l} \otimes \mathbb{E}, \omega_{Y}^{-k} \otimes \mathbb{E}\right)
$$

is an isomorphism.

Since $\mathbb{E}$ is a classical generator for $D^{b}\left(\operatorname{coh}\left(\mathcal{O}_{Y}\right)\right)$ it suffices to prove the result with $\omega_{Y}^{-k} \otimes \mathbb{E}$ and $\omega_{Y}^{l} \otimes \mathbb{E}$ replaced by $\mathbb{E}$. Then the statement is obvious.

Lemma A.2. Consider $A(\mathbb{E}) \otimes A(\mathbb{E})$ as $A(\mathbb{E})$-bimodule with the outer bimodule structure. We have an equality of $A(\mathbb{E})$-bimodules

$$
B(\mathbb{H})_{1}=\operatorname{RHom}_{A(\mathbb{E})^{e}}(A(\mathbb{E}), A(\mathbb{E}) \otimes A(\mathbb{E}))[2]
$$

where on the righthandside the $A(\mathbb{E})$-bimodule structure comes from the surviving inner bimodule structure on $A(\mathbb{E}) \otimes A(\mathbb{E})$. In other words $B(\mathbb{H})_{1}$ is a shifted inverse dualizing complex of $A(\mathbb{E})([12, \S 3.3])$.

Proof. We have to compute

$$
\operatorname{RHom}_{\operatorname{End}(\mathbb{E})^{e}}(\operatorname{End}(\mathbb{E}), \operatorname{End}(\mathbb{E}) \otimes \operatorname{End}(\mathbb{E}))
$$

Working on $Y \times Y$ we have

$$
\operatorname{End}(\mathbb{E})^{e} \cong \operatorname{End}\left(\mathbb{E} \otimes \mathbb{E}^{*}\right)
$$

It is easy to see that $\mathbb{E} \otimes \mathbb{E}^{*}$ is a classical generator for $D^{b}(\operatorname{coh}(Y \times Y))$ (e.g. because it is derived from an exceptional collection, or else invoke [5]). Under the ensuing derived equivalence $D^{b}\left(\operatorname{End}\left(\mathbb{E} \otimes \mathbb{E}^{*}\right)\right) \cong D^{b}(\operatorname{coh}(Y \times Y))$ it is easy to see that $\operatorname{End}(\mathbb{E})$ corresponds to $\mathcal{O}_{Y}$ and $\operatorname{End}(\mathbb{E}) \otimes \operatorname{End}(\mathbb{E})$ to $\mathbb{E} \otimes \mathbb{E}^{*}$.

We now have to compute

$$
\operatorname{RHom}_{Y \times Y}\left(\mathcal{O}_{\Delta}, \mathbb{E} \otimes \mathbb{E}^{*}\right)
$$

where $\Delta$ is the diagonal. We use the well-known formula

$$
\mathrm{RHom} \operatorname{\mathcal {O}}_{Y \times Y}\left(\mathcal{O}_{\Delta}, \mathcal{O}_{Y \times Y}\right)=\omega_{\Delta}^{-1}[-2]
$$

Thus

$$
\begin{aligned}
& \operatorname{RHom}_{Y \times Y}\left(\mathcal{O}_{\Delta}, \mathbb{E} \otimes \mathbb{E}^{*}\right)=R \Gamma\left(Y \times Y, \mathrm{RHom}_{Y \times Y}\left(\mathcal{O}_{\Delta}, \mathcal{O}_{Y \times Y}\right) \stackrel{L}{\otimes} Y \times Y \mathbb{E} \otimes \mathbb{E}^{*}\right) \\
& =R \Gamma\left(Y \times Y, \omega_{\Delta}^{-1} \stackrel{L}{\otimes}_{Y \times Y} \mathbb{E} \otimes \mathbb{E}^{*}\right)[-2] \\
& =\operatorname{RHom}_{Y}\left(\mathbb{E}, \omega_{Y}^{-1} \otimes \mathbb{E}\right)[-2]
\end{aligned}
$$


Lemma A.3. Assume that $B$ is an $\mathbb{N}$-graded ring such that $\operatorname{dim} B_{i}<\infty$ for all $i$. Then

$$
\text { gl } \operatorname{dim} B_{0} \leq \mathrm{gl} \operatorname{dim} B
$$

If the category of graded finite length B-modules is Ext-finite and has a Serre functor given by $?(d)[n]$ for $d \neq 0$ then

$$
\text { gl } \operatorname{dim} B_{0}<\operatorname{gl} \operatorname{dim} B=n
$$

Proof. Let $S, T$ be simple $B_{0}$-modules which we view as $B$-modules concentrated in degree zero. The part of degree zero of a graded projective $B$-resolution of $S$ is a projective $B_{0}$-resolution of $S$. This implies the first inequality. We also get

$$
\operatorname{Ext}_{B_{0}}^{i}(S, T)=\operatorname{Ext}_{B}^{i}(S, T)_{0}
$$

Assume now that the category of graded finite length $B$-modules is Ext-finite and has a Serre functor given by ? $(d)[n]$ for $d \neq 0$. Then it is standard that $\mathrm{gl} \operatorname{dim} B=n$. For the second inequality we note that $\operatorname{Ext}_{B}^{n}(S, T)_{0}=\operatorname{Hom}_{B}(T, S(d))_{0}^{*}=0$.

Proof of Theorem 4.2.1. Since $A(\mathbb{E})$ is finite dimensional and has finite global dimension the same is true for $A(\mathbb{E})^{e}$. Hence $A(\mathbb{E})$ is perfect as a bimodule and as such homologically smooth.

By Lemmas A.1,A.2 we obtain that $B(\mathbb{H})$ is a (graded) 3-Calabi-Yau completion of $A(\mathbb{E})$ (see $[12, \S 4]$ ). By [12, Thm 4.8] we obtain that $B(\mathbb{H})$ is 3 -Calabi-Yau and the proof shows that the Serre functor is given by ?(-1). By Lemma A.3 we then find that $\operatorname{gl} \operatorname{dim} A(\mathbb{E}) \leq 2$. We may then use $[12$, Thm 6.10$]$ to obtain that $B(\mathbb{H})$ is quasi-isomorphic to a Ginzburg algebra derived from a super potential of degree one. Since $B(\mathbb{H})$ is concentrated in degree zero it is also a Jacobian algebra.

To prove that $B(\mathbb{H})$ is almost Azumaya we note that $B(\mathbb{H})$ is the pushforward of an Azumaya algebra on the canonical bundle of $Y$. This easily yields the desired result.

\section{REFERENCES}

[1] C. Amiot and S. Oppermann, Cluster equivalence and graded derived equivalence, arXiv:1003.4916.

[2] R. Bocklandt, Graded Calabi Yau algebras of dimension 3, J. Pure Appl. Algebra 212 (2008), no. 1, 14-32.

[3] R. Bocklandt, T. Schedler, and M. Wemyss, Superpotentials and higher order derivations, J. Pure Appl. Algebra 214 (2010), no. 9, 1501-1522.

[4] A. Bondal and A. Polishchuk, Homological properties of associative algebras: the method of helices, Russian Acad. Sci. Izv. Math 42 (1994), 219-260.

[5] A. Bondal and M. Van den Bergh, Generators and representability of functors in commutative and noncommutative geometry, Mosc. Math. J. 3 (2003), no. 1, 1-36, 258.

[6] T. Bridgeland and D. Stern, Helices on del Pezzo surfaces and tilting Calabi-Yau algebras, arXiv:0909.173.

[7] A. B. Buan, O. Iyama, I. Reiten, and D. Smith, Mutation of cluster-tilting objects and potentials, arXiv:0804.3813.

[8] F. Cachazo, S. Katz, and C. Vafa, Geometric transitions and $\mathcal{N}=1$ quiver theories, arXiv:hep-th/0108120.

[9] W. Crawley-Boevey and M. P. Holland, Non-commutative deformations of Kleinian singularities, Duke Math. J. 92 (1998), 605-635.

[10] H. Derksen, J. Weyman, and A. Zelevinsky, Quivers with potentials and their representations. I. Mutations, Selecta Math. (N.S.) 14 (2008), no. 1, 59-119.

[11] O. Iyama and I. Reiten, Fomin-Zelevinsky mutation and tilting modules over Calabi-Yau algebras, Amer. J. Math. 130 (2008), no. 4, 1087-1149. 
[12] B. Keller, Deformed Calabi-Yau completions, J. Reine Angew. Math. 654 (2011), 125-180, With an appendix by Michel Van den Bergh.

[13] B. Keller and D. Yang, Derived equivalences from mutations of quivers with potential, to appear in Advances in Mathematics.

[14] K. Kurano and S. Nishi, Gorenstein isolated quotient singularities of odd prime dimension are cyclic, arXiv:0903.3270v1.

[15] H. Lenzing, Nilpotente Elemente in Ringen von endlicher globaler Dimension, Math. Z. 108 (1969), 313-324.

[16] H. Minamoto, Ampleness of two-sided tilting complexes, to appear.

[17] H. Minamoto and I. Mori, Structures of AS-regular algebra, to appear.

[18] A. Quintero-Velez and A. Boer, Noncommutative resolutions of ADE fibered Calabi-Yau threefolds, arXiv:0806.3177.

[19] I. Reiten and M. Van den Bergh, Two-dimensional tame and maximal orders of finite representation type, vol. 80, Memoirs of the AMS, no. 408, Amer. Math. Soc., July 1989.

[20] E. Segal, The $A_{\infty}$ deformation theory of a point and the derived categories of local CalabiYaus, J. Algebra 320 (2008), no. 8, 3232-3268.

[21] M. Van den Bergh, Calabi-Yau algebras and superpotentials, arXiv:1008.0599v1 [math.KT].

[22] _ Non-commutative crepant resolutions, The legacy of Niels Henrik Abel (Berlin), Springer, Berlin, 2004, pp. 749-770.

E-mail address: louicious@mac.com, michel.vandenbergh@uhasselt.be

Departement WNi, Universiteit Hasselt, 3590 Diepenbeek, Belgium. 